\title{
Sarcoma histiocítico de intestino delgado. Reporte de un caso y revisión de la literatura
}

\author{
Alejandro Avilés-Salas ${ }^{1}$, María de Lourdes Peña-Torresa ${ }^{a}$, \\ Angel Molina-Cruz ${ }^{1}$, Silvia Rivas-Vera ${ }^{2}$.
}

\author{
Histiocytic sarcoma of the small \\ intestine. Report of one case
}

Histiocytic sarcoma (HS) is a rare malignant neoplasm of the lymphohematopoietic system, that occurs in lymph nodes, skin and at extranodal sites, particularly the gastrointestinal tract. Although it shows characteristics histological and immunohistochemical features, it may be misdiagnosed. We report a 67 year-old female patient presenting with colicky abdominal pain and vomiting. A CT scan of the abdomen revealed a tumor in the ileum, that was surgically removed. On pathology, the neoplastic cells displayed large abundant eosinophilic cytoplasm, with bizarre-shaped nuclei, that expressed CD 45, CD 68 and lisozyme. The diagnosis of HS requires the use of a panel of immunohistochemical markers and may be supported by ultrastructural findings (Rev Méd Chile 2009; 137: 269-74).

(Key words: Histiocytoma, malignant fibrous; Ileum; Sarcoma)
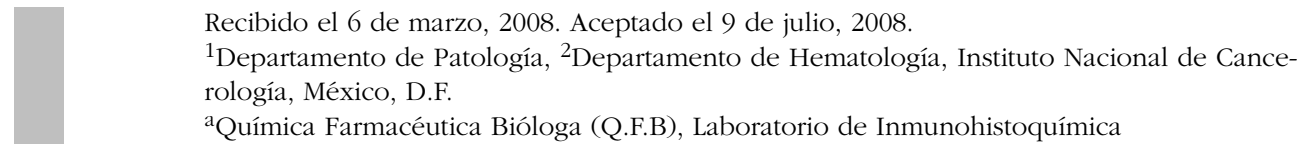

$\mathrm{L}$ as células dendríticas/histiocíticas son un grupo heterogéneo del sistema inmunológico presentes en órganos linfoides y no linfoides ${ }^{1}$. La Organización Mundial de la Salud clasifica las neoplasias de células dendríticas/histiocíticas en: sarcoma histiocítico (SH), histiocitosis de células de Langerhans, sarcoma de células de Langerhans, sarcoma de células dendríticas interdigitantes, sarcoma de células dendríticas foliculares y sarcoma de células dendríticas sin otra especificación ${ }^{2}$. El SH lo describió inicialmente Rappaport como una neoplasia maligna de estirpe linfoide ${ }^{3}$; sin embargo, con el uso de nuevos marcadores de

$\overline{\text { Correspondencia a: Dr. Alejandro Avilés Salas. Departamen- }}$ to de Patología, Instituto Nacional de Cancerología. Av. San Fernando 22, Sección XVI. Tlalpan. 14080, México, D.F. E mail: alejandroaviles2001@yahoo.com inmunohistoquímica y la aplicación de técnicas de biología molecular, se ha demostrado que la gran mayoría de los casos diagnosticados como linfoma histiocítico corresponden a linfomas no Hodgkin (LNH), predominantemente linfomas difusos de células grandes B y linfomas anaplásicos ${ }^{4,5}$. El SH es una neoplasia rara que afecta con mayor frecuencia a adultos. Aproximadamente una tercera parte de los casos se han ubicado en linfonodos, una tercera parte en la piel y el resto en sitios extraganglionares $^{2}$. Informamos un caso de $\mathrm{SH}$ primario del íleon y revisamos la literatura sobre el tema.

\section{Caso Clínico}

Paciente femenina de 67 años de edad con diabetes mellitus tipo 2, que inició su padecimiento 18 
meses previos a su hospitalización con vómitos, dolor abdominal e intolerancia a alimentos sólidos, por lo cual fue referida al Instituto Nacional de Cancerología. A su ingreso se encontró deshidratada, con facies de dolor, posición antiálgica, índice Karnofsky de 80\% y ECOG de 1. En la exploración física el abdomen estaba distendido y se palpó un tumor en el hemiabdomen izquierdo, sin hepatoesplenomegalia ni adenopatías. Se realizó tomografía axial computarizada (TC) abdominopélvica que mostró un tumor de aspecto lobulado en el tercio distal del íleon, con engrosamiento de la pared, estenosis de la luz y extensión al mesenterio (Figura 1). Se programó para laparotomía exploradora con resección del segmento de íleon y anastomosis término-terminal. El diagnóstico transoperatorio fue de linfoma. Para el estudio anatomopatológico se recibió un segmento de íleon de $18 \mathrm{~cm}$ con tumor de aspecto nodular, ulcerado, de bordes irregulares que midió $8 \times 8 \times 6 \mathrm{~cm} \mathrm{e}$ infiltraba hasta la serosa. En el estudio histológico se observó una neoplasia maligna constituida por células grandes, dispuestas en forma difusa. Las células tenían núcleos ovoides, ocasionalmente hendidos, con cromatina fina y nucléolo aparente. Había atipia y pleomorfismo marcados; así como células binucleadas. Una característica sobresaliente fue el fondo rico en linfocitos, neutrófilos, eosinófilos y ocasionales células plasmáticas (Figura 2a). Se realizó un estudio de inmunohistoquími- ca con el método estándar streptavidina-biotinaperoxidasa (Tabla 1). Las células neoplásicas expresaron CD 45, CD 68 y lisozima, lo cual apoyó el diagnóstico de $\mathrm{SH}$ (Figura 2b). Los linfocitos reactivos fueron predominantemente de estirpe T. Se realizó un estudio de microscopia electrónica en el cual se observaron células con núcleo ovoide de contorno lobulado, cromatina granular y nucléolo aparente. El citoplasma de las células neoplásicas contenía retículo endoplásmico rugoso, lisosomas y mitocondrias (Figura 3). La paciente evolucionó satisfactoriamente y egresó en enero de 2007. En mayo de 2007 se practicó PET-CT de cuerpo entero que informó actividad tumoral en ganglios mesentéricos, retroperitoneales y un tumor abdominal multilobulado localizado entre el recto anterior derecho y el peritoneo parietal, sugerentes de recurrencia. Se programó laparotomía exploradora con resección en bloque de implante de pared abdominal y segmento del íleon con disección selectiva de ganglios linfáticos retroperitoneales. El estudio histológico del segmento de intestino delgado y de los ganglios linfáticos exhibió características morfológicas y perfil de inmunohistoquímica similares a las descritas en el tumor primario. Se decidió administrar 6 ciclos de quimioterapia con esquema de CHOP (ciclofosfamida, doxorrubicina, vincristina y prednisona). Actualmente, la paciente muestra una respuesta clínica completa.

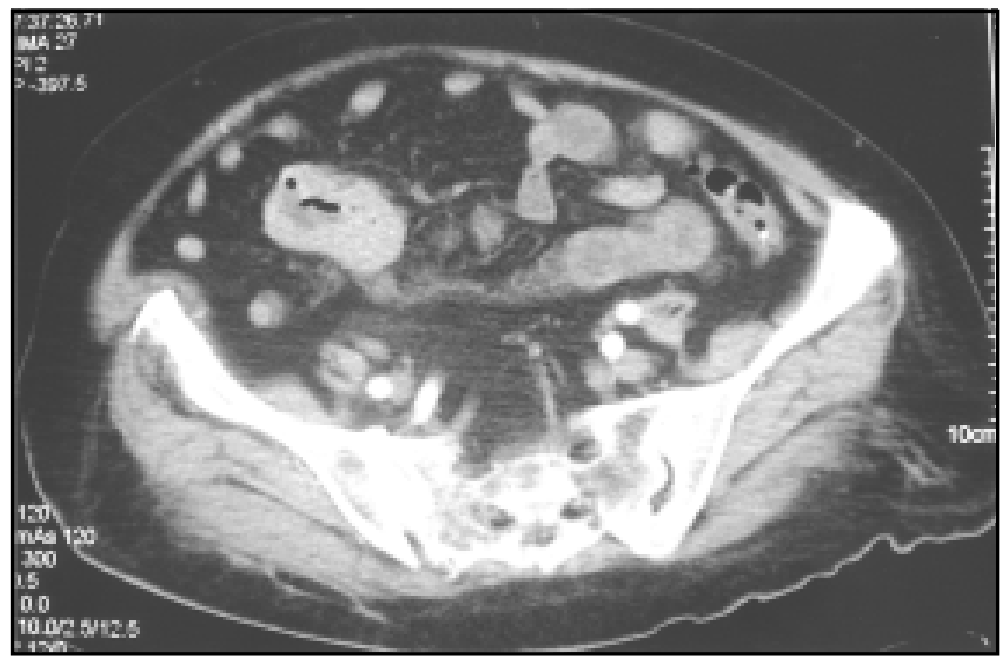

Figura 1. TC abdominopélvica que muestra lesión sólida del tercio distal del íleon, con engrosamiento de la pared y extensión al mesenterio. 

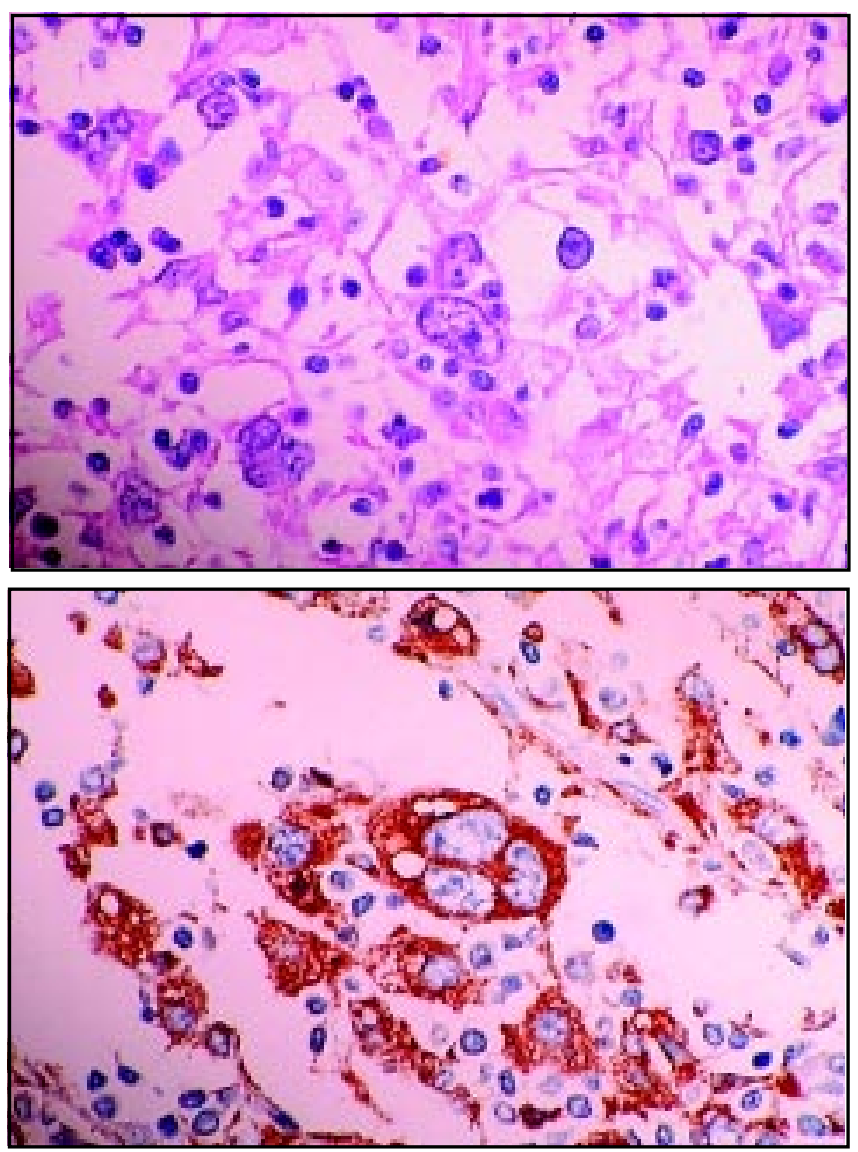

Figura 2. a) Las células neoplásicas son ovoides, alternan con linfocitos y células plasmáticas y muestran atipia celular y pleomorfismo (Hematoxilina-eosina, 40X). b) Expresión de CD 68 en células neoplásicas (Técnica de inmunohistoquímica, 40X).

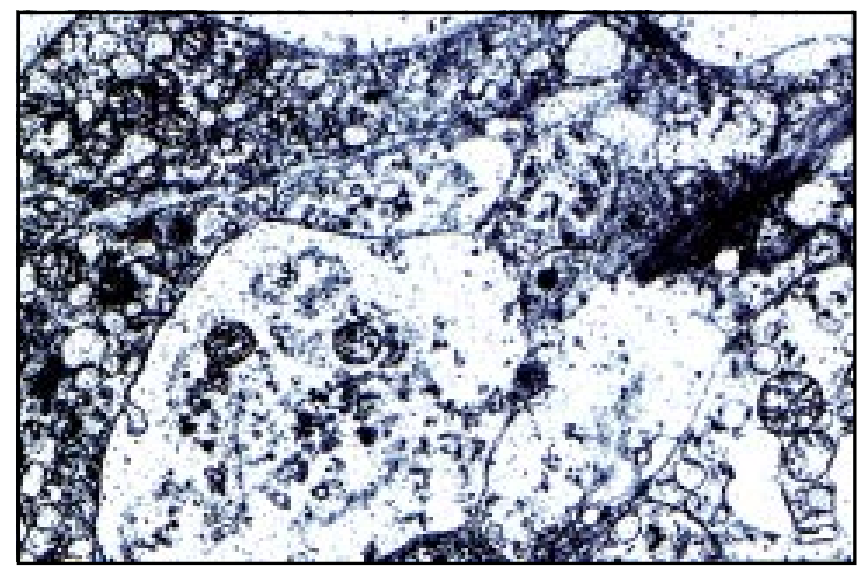

Figura 3. A nivel estructural las células neoplásicas tienen núcleo ovoide, retículo endoplásmico rugoso y abundantes mitocondrias y lisosomas (Técnica de microscopia electrónica, 12000X). 
Tabla 1. Anticuerpos utilizados en el estudio de inmunohistoquímica

\begin{tabular}{|llll|}
\hline Anticuerpo & Clona & Dilución & Marca de fábrica \\
\hline Vimentina & V9 & $1: 3000$ & BIOGENEX \\
CD 45 & LCA 88 & $1: 1000$ & BIOGENEX \\
CD 68 & KP1 & $1: 2000$ & DAKO \\
Lisozima & - & $1: 8000$ & DAKO \\
CD 21 & 2G9 & $1: 300$ & BioSB \\
CD 23 & 1B12 & $1: 50$ & Cell-Marque \\
CD 35 & Ber-MAC-DRC & $1: 10$ & DAKO \\
CD 20 & L26 & $1: 80$ & BIOGENEX \\
CD 45Ro & UCHL-1 & $1: 600$ & DAKO \\
CD 15 & BRA4F1 & $1: 100$ & BIOGENEX \\
CD 30 & HRS-4 & $1: 400$ & BIOGENEX \\
Citoqueratina & AE1/AE3 & $1: 200$ & DAKO \\
Proteína S-100 & 15E2E2 200 \\
Actina & HHF35 & $1: 7500$ & BIOGENEX \\
ALK-1 & ALK-1 & $1: 500$ & DAKO \\
Melanoma & HMB45 & $1: 50$ & Cell-Marque \\
c-Kit & - & $1: 100$ & BIOGENEX \\
EMA & Mc5 & $1: 1000$ & DAKO \\
\hline
\end{tabular}

DisCUSIÓN

El $\mathrm{SH}$ se ha reportado en un amplio rango de edad; sin embargo, la mayoría de los casos ocurren en adultos, con una mediana de 46 años $^{6,7}$. Se ha ubicado en diferentes sitios extraganglionares, que incluyen piel, pulmón, cavidad nasal, paladar, hueso, tracto gastrointestinal, bazo, hígado, tejidos blandos y sistema nervioso central $^{6-12}$. El presente caso se suma a los previamente descritos en el tracto gastrointestinal, que incluyen localizaciones en el esófago ${ }^{13}$, estómago ${ }^{14}$, intestino delgado ${ }^{6-8,15-17}$, colon $^{8,9}$, recto y ano $^{6}$. Las características clínicas se resumen en la Tabla 2.

La etiología del $\mathrm{SH}$ se desconoce. Se han informado casos asociados con linfomas, leucemias, mielodisplasia, mielofibrosis y tumores de células germinales del mediastino, en particular teratomas malignos con o sin diferenciación a tumor de senos endodérmicos. Se ha sugerido que el $\mathrm{SH}$ se puede originar de células germinales pluripotenciales, dada la asociación entre estas neoplasias y la capacidad in vitro del teratocarci- noma de diferenciarse a líneas hematopoyéti$\operatorname{cas}^{2,7,18}$.

Desde el punto de vista morfológico es un tumor constituido por células grandes redondas $\mathrm{u}$ ovoides, no cohesivas, dispuestas en forma difusa. Las células neoplásicas tienen un citoplasma eosinófilo amplio, bordes celulares poco definidos, núcleo ovoide con cromatina finamente granular o vesicular y nucléolo aparente. La atipia y pleomorfismo celular usualmente son moderados; sin embargo, se pueden observar células binucleadas tipo Reed-Sternberg o células gigantes multinucleadas con hemofagocitosis. El número de mitosis es variable, observándose un mayor número en los casos con atipia citológica marcada. Una característica de este tumor es la presencia de linfocitos predominantemente de estirpe T, células plasmáticas e histiocitos ${ }^{2,6-9}$.

Las células del SH son positivas a marcadores de histiocitos, que incluyen CD 68, lisozima, CD 11c y CD 14 y de forma ocasional proteína S$100^{2,6-9}$. Pileri y cols, utilizando un panel de seis anticuerpos (CD 21, CD 23, CD 35, CD 68, CD 1a y lisozima) pudieron clasificar de forma correcta 
Tabla 2. C aracterísticas clínicas de SH del tracto gastrointestinal. (No se incluyen los casos localizados en hígado ni páncreas)

\begin{tabular}{|c|c|c|c|c|c|c|c|}
\hline Referencia & Caso & $\begin{array}{l}\text { Edad } \\
\text { (años) }\end{array}$ & Género & Localización & Tratamiento & $\begin{array}{l}\text { Seguimiento } \\
\text { (meses) }\end{array}$ & Evolución \\
\hline \multirow[t]{5}{*}{ Hornick $^{6}$} & 1 & 89 & M & Colon/estómago & Cirugía & 5 & MPE \\
\hline & 2 & 40 & $\mathrm{~F}$ & Recto & Cirugía & ND & ND \\
\hline & 3 & 58 & M & ID (îleon) & Cirugía & ND & ND \\
\hline & 4 & 27 & $\mathrm{~F}$ & Recto & Cirugía & 120 & VSE \\
\hline & 5 & 40 & $\mathrm{~F}$ & Ano & Cirugía & ND & ND \\
\hline \multirow[t]{4}{*}{ Pileri $^{7}$} & 6 & 56 & M & ID & $C+Q$ & ND & MPE \\
\hline & 7 & 46 & F & ID & Cirugía & ND & ND \\
\hline & 8 & ND & M & ID & ND & ND & ND \\
\hline & 9 & ND & M & ID & ND & ND & ND \\
\hline \multirow[t]{6}{*}{ Copie-Bergman $^{8}$} & 10 & 26 & $\mathrm{~F}$ & ID & ND & ND & ND \\
\hline & 11 & 45 & $\mathrm{~F}$ & ID & ND & ND & ND \\
\hline & 12 & 54 & M & ID & ND & ND & ND \\
\hline & 13 & 56 & M & ID & ND & ND & ND \\
\hline & 14 & 36 & M & ID (duodeno) & ND & ND & ND \\
\hline & 15 & 69 & M & Colon & ND & ND & ND \\
\hline \multirow[t]{2}{*}{$\operatorname{Vos}^{9}$} & 16 & 20 & $\mathrm{~F}$ & Colon & Quimioterapia & 15 & MPE \\
\hline & 17 & 55 & M & Colon & Cirugía & 2 & MPE \\
\hline $\mathrm{Seo}^{13}$ & 18 & 56 & $\mathrm{~F}$ & Esófago & Quimioterapia & 5 & MPE \\
\hline Alvaro $^{14}$ & 19 & 52 & $\mathrm{~F}$ & Estómago & $C+Q$ & 18 & VSE \\
\hline \multirow[t]{2}{*}{ Miettinen $^{15}$} & 20 & 52 & $\mathrm{~F}$ & ID & $C+Q$ & 16 & MPE \\
\hline & 21 & 58 & M & ID (îleon) & $C+Q$ & 12 & VSE \\
\hline \multirow[t]{2}{*}{ Milchgrub $^{16}$} & 22 & 60 & M & ID (îleon) & $C+Q$ & 24 & VSE \\
\hline & 23 & 63 & M & ID (îleon) & $C+Q$ & 51 & MPE \\
\hline Watanabe $^{17}$ & 24 & 45 & M & ID (íleon) & $C+Q$ & 3 & MPE \\
\hline Avilés-Salas & 25 & 67 & $\mathrm{~F}$ & ID (îleon) & $C+Q$ & 12 & VSE \\
\hline
\end{tabular}

F: femenino, M: masculino, ID: intestino delgado, C + Q: cirugía + quimioterapia, VCE : vivo con enfermedad, VSE: vivo sin enfermedad, MPE: muerto por enfermedad, ND: no documentado.

93\% de los casos de tumores de células dendríticas/histiocíticas?.

Por ultraestructura las células del SH muestran abundante citoplasma con numerosos lisosomas. No hay complejos de unión, tonofilamentos, microfilamentos, interdigitaciones citoplásmicas ni gránulos de Birbeck ${ }^{2,7}$.

El diagnóstico diferencial debe incluir neoplasias hematológicas y no hematológicas. Dentro del primer grupo se deben considerar los diagnósticos de linfoma de Hodgkin, linfoma anaplásico, sarcoma de células dendríticas interdigitantes y sarcoma de células dendríticas foliculares. En nuestro caso, en base al aspecto morfológico se consideró como diagnóstico inicial un linfoma anaplásico versus linfoma de Hodgkin; sin embargo, los marcadores de inmunohistoquímica (CD 15, CD 30; CD 20, CD 3 y ALK-1) fueron negativos en las células neoplásicas y positivas para CD 68, CD 45 y lisozima. Otros diagnósticos no hematológicos que deben ser tomados en cuenta, sobre todo cuando la localización es extraganglionar, incluyen: tumor del estroma gastrointestinal, tumor maligno de vaina de nervio periférico, histiocitoma fibroso maligno, pseudotumor inflamatorio, carcinoma tipo linfoepitelioma y melanoma ${ }^{2,6,7}$.

El SH es una neoplasia con un comportamiento usualmente agresivo, el cual se ha manejado con 
varios tratamientos incluyendo cirugía, radioterapia, quimioterapia y combinaciones de éstos. En pacientes con enfermedad localizada la resección quirúrgica es la modalidad más utilizada, siendo los resultados variables, dependiendo la extensión de la lesión. La recurrencia local después de la resección inicial se puede manejar con cirugía. En nuestro caso la paciente se trató inicialmente con cirugía, mostrando recurrencia a los tres meses de seguimiento, lo cual requirió de manejo quirúrgico más quimioterapia, con buena respuesta. Aun cuando no existe un tratamiento estándar para esta neoplasia, la asociación de cirugía más quimioterapia fue de utilidad en nuestra paciente, ya que aproximadamente $60 \%$ de los pacientes mueren por progresión de la enfermedad $2,7,8$.

\section{REFERENCIAS}

1. Kairouz S, Hashash J, Kabbara W, Mchaylen W, Tabara A. Dendritic cell neoplasms: an overview. Am J Hematol 2007; 82: 924-8.

2. Weiss L, Grogan TM, Müller-Hermelink HK, Stein H. Histiocytic sarcoma. En: Jaffe ES, Harris NL, Stein H, Vardiman JW, ed. Pathology and Genetic of Tumours of Haematopoietic and Lymphoid Tisues. Lyon: IARC Press, 2001; 273-90.

3. RAPPAPORT H. Tumors of Haematopoietic System. Section III, Fascicle 8. Washington, DC: Armed Forces Institute of Pathology, 1996.

4. Lukes RJ, Taylor CR, Chir B. A morphologic and immunologic surface marker study of 299 cases of non-Hodgkin lymphomas and related leukemias. Am J Pathol 1978; 90: 461-86.

5. PINkUS GS, SAID JW. Characterization of nonHodgkin's lymphomas using multiple cell markers. Immunologic, morphologic and cytochemical studies of 72 cases. Am J Pathol 1978; 94: 349-80.

6. Hornick JL, Jaffe ES, Fletcher CD. Extranodal histiocytic sarcoma. Clinicopathologic analysis of 14 cases of a rare epithelioid malignancy. Am J Surg Pathol 2004; 28: 1133-44.

7. Pileri Sa, Grogan TM, Harkis NL, Banks P, Campo E, Chan JKC ET AL. Tumour of histiocytes and accessory dendritic cells: an immunohistochemical approach to classification from the International Lymphoma Study Group based on 61 cases. Histopathology 2002; 41: 1-29.

8. Copie-Bergman C, Wotherspoon AC, norton AJ, Diss TC, IsAAACSON PG. The histiocytic lymphoma. A morphologic, immunohistochemical and molecular genetic study of 13 cases. Am J Surg Pathol 1998; 22: 1386-92.

9. Vos Ja, Abbondanzo SL, Barekman CL, Andriko JW, Miettinen M, Aguilera NS. Histiocytic sarcoma: a
En conclusión, el $\mathrm{SH}$ es un tumor poco frecuente y aun cuando tiene características morfológicas, inmunohistoquímicas y ultraestructurales, su diagnóstico no es sencillo, y generalmente incluye dentro del diagnóstico diferencial a neoplasias hematológicas y no hematológicas. Al momento no existe consenso respecto al tratamiento estándar; sin embargo, cuando la lesión está localizada, se recomienda como primera opción terapéutica la resección quirúrgica. El comportamiento biológico del $\mathrm{SH}$ es agresivo, con un alto índice de recurrencia local, aun cuando se requiere un mayor número de casos para establecer factores estadísticamente importantes asociados al pronóstico.

study of five cases including the histiocyte marker CD163. Mod Pathol 2005; 18: 693-704.

10. Buonocore S, Valente AL, Nightingale D, Bogart J, SouID AK. Histiocytic sarcoma in a 3-year-old male: a case report. Pediatrics 2005; 116: 322-5.

11. Sun W, Nordberg ML, Fowler MR. Histiocytic sarcoma involving the central nervous system. Clinical, immunohistochemical, and molecular genetic studies of a case with review of the literature. Am J Surg Pathol 2003; 27: 258-65.

12. Cao M, Eshoa C, Schultz C, Black J, Zu Y, Chang CC. Primary central nervous system histiocytic sarcoma with relapse to mediastinum. A case report and review of the literature. Arch Pathol Lab Med 2007; 131: 301-5.

13. Seo IS, Henley JD, Min KW, Yum MN. True histiocytic lymphoma of esophagus in an HIV-positive patient: an ultrastructural study. Ultrastruct Pathol 1999; 23: 333-9.

14. Alvaro T, Bosch R, Salvado MT, Piris MA. True histiocytic lymphoma of the stomach associated with low-grade Bcell mucosa-associated lymphoid tissue (MALT)-type lymphoma. Am J Surg Pathol 1996; 20: 1406-11.

15. Miettinen M, Fletcher C, Lasota J. True histiocytic lymphoma of small intestine. Analysis of two S-100 protein-positive cases with features of interdigitating reticulum cell sarcoma. Am J Clin Pathol 1993; 100: 285-92.

16. Milchgrub S, Kamel OW, Wiley E, Vuitch F, Cleary ML, WARNKE RA. Malignant histiocytic neoplasma of the small intestine. Am J Surg Pathol 1992; 16: 11-20.

17. Watanabe S, Hirota T, Shimosato Y, Ito A, Zeze F, Hojo $\mathrm{K}$. Unusual histiocytic tumor of the small intestine. Hum Pathol 1980; 11: 289-93.

18. Fununaga M, Kato H. Histiocytic sarcoma associated with idiopathic myelofibrosis. Arch Pathol Lab Med 2004; 128: 1167-70. 